\title{
Consultation on SNH draft amphibian and reptile strategy
}

\author{
D.C. McNeill \\ Kelvin Building, University of Glasgow, Glasgow, G12 8QQ \\ E-mail: Deborah.Mcneill@glasgow.ac.uk
}

\section{INTRODUCTION}

The conference Amphibians and Reptiles of Scotland: current research and future challenges took place on Saturday 9th June 2018 as part of the Glasgow Science Festival. On behalf of Scottish Natural Heritage (SNH), Rob Raynor presented a talk on the SNH draft amphibian and reptile strategy for Scotland. The threats, opportunities and challenges for Scottish herpetological conservation were explored, with a recognition of the importance of effective partnership with relevant organisations to meet conservation objectives. This was followed by three facilitated workshops to enable discussion and comment on the draft strategy.

An audience of over 80 participants took part with representation from key herpetological and conservation organisations, research staff and students, consultancies and interested public. Fifty responses were collated by facilitators/scribes.

\section{THE WORKSHOPS}

Participants attended one of three workshops of their choosing, each on one of the draft strategy's priorities:

1. To improve people's understanding and appreciation of amphibians and reptiles, reduce persecution and build public support for conserving them.

2. To address the range of ecological threats and pressures affecting Scottish amphibians and reptiles.

3. To improve the quality of information we hold on the distribution and status of amphibians and reptiles through appropriate surveillance and monitoring.

The following questions were addressed:

1. Are these the right priorities?

2. Can we rank them?

3. Are there any key omissions? (These need to be justified.)

4. How deliverable are they and who would be responsible for their delivery?

\section{WORKSHOP OUTCOMES}

Each of the three workshops featured a list of priorities, which are shared below, ranked in order from most important to of least concern by all participants. A summary of comments pertaining to these priorities was collated.

\section{Workshop 1}

Participants ranked the following first three points as equally important. It was also deemed that there was overlap between the questions and as such, they were commented on together.

1a) Increase the number of people actively involved in conservation projects of benefit to amphibians and reptiles.

1b) Increase public understanding and appreciation of amphibians and reptiles.

1c) Promote practical conservation measures involving volunteers and members of the local community to benefit amphibians and reptiles on land owned or managed by Non-Governmental Organisations (NGOs), local authorities and statutory undertakers and on private estates.

Discussion: Participants raised resource issues: for example, the shortage of local authority biodiversity officers needed to develop Local Biodiversity Action Plans (LBAPs), and the comparative shortage of funds for conservation work (in Scotland compared with England). Points on public understanding stressed the need to choose audiences carefully, to use evidence-based approaches, and to be subtle in approaches aimed at gaining public support for amphibians and reptiles.

2a) Promote collaboration amongst conservation organisations to identify synergies between unrelated species-focused projects and strategies of potential benefit to amphibians and reptiles, as illustrated by the Roots of Rockingham Project (Nature Back From The Brink, 2018) and the SNH Pollinator Strategy 2017-2027 (SNH, 2017).

Discussion: Comments supported the idea that strategic priorities could provide a clearer focus for NGO activities. However, there was concern over inconsistency and frequent changes in plans and reporting requirements, especially for LBAPs. 
2b) Promote more widespread adoption of amphibian-friendly measures to reduce mortality associated with roads and road drains.

Discussion: This aim was widely agreed, with comments emphasising the need for improved legal protection of amphibians and incorporation of amphibian mitigation into the planning process. Common toad (Bufo bufo) patrols provide effective public engagement as well as protection.

3) Reduce persecution of European adders (Vipera berus) and slow-worms (Anguis fragilis).

No comments were recorded.

Additional comments: Participants emphasised the political battle needed to improve amphibian and reptile conservation in Scotland; the lack of attendance by any Member of the Scottish Parliament (MSP) Species Champions or their staff was telling.

\section{Workshop 2}

1) Review how best to optimise habitat connectivity in both urban and rural areas in relation to important amphibian and reptile sites, and work with key stakeholders to integrate their needs into infrastructure schemes and initiatives.

2) Promote the inclusion of more focused habitat management options designed to benefit amphibians and reptiles within future forestry and agri-environment funding schemes.

Discussion: It was felt that current government priorities for forestry and agricultural development are a potential threat to amphibians and reptiles unless their needs are carefully considered. Agrienvironment schemes rarely focus on amphibians and reptiles, but this could change in well-chosen areas.

3) Ensure that key best practice guidance is up-todate and, if necessary, revised with an appropriate Scottish emphasis.

Discussion: Participants would welcome an easily accessible source of comprehensive and up-to-date best practice guidelines hosted on e.g. the $\mathrm{SNH}$ website. The question was asked as to whether there could be better connectivity with biodiversity guidelines for Sustainable Drainage Systems (SuDS) and urban development, produced by SNH, the Scottish Environment Protection Agency (SEPA) and Scottish Water.

4) Improve our understanding of how upland land management activities such as spring muirburn may impact on reptiles and amphibians and develop appropriate mitigation guidance as appropriate.
5) Assess benefits of green infrastructure to reptiles and amphibians and how predicted effects of climate change can be mitigated through these and other measures to improve habitat connectivity in general.

Discussion: One example explored was a targeted approach to communicating potential biodiversity benefits of SuDS to developers.

6) Promote awareness of disease risks and remain vigilant, ensuring an appropriate and rapid response in accordance with the Invasive Non-Native Species (INNS) code of practice (Scottish Government, 2012) to suspected outbreaks of harmful infectious diseases.

7) Build on previous studies to better understand how climate change might affect Scotland's amphibians and reptiles.

8) Promote a responsible attitude towards species translocations generally, particularly in relation to the assisted movement of fish between water bodies.

9) Remain vigilant and ensure an appropriate and rapid response in accordance with the INNS code of practice to reports of any new non-native species of amphibian or reptile in Scotland. This includes ongoing monitoring of existing non-native populations and the identification of potential new threats.

10) Promote the guidance on biosecurity provided by Amphibian and Reptile Groups of U.K. (ARG UK, 2017).

Additional comments: Participants discussed the need to identify and address current knowledge gaps. A better understanding of the effectiveness of herpetological sites that are currently afforded protection will offer insights into e.g. where resources might be best utilised for improvement. Utilising tools such as GIS mapping of key populations can feed into predictive mapping to identify potential new sites, underpinned by a programme of baseline population surveys. One suggestion was to link the strategy to appropriate regulatory bodies so $\mathrm{SNH}$ is not solely responsible for guidance and regulation.

\section{Workshop 3}

1) Increase collaboration between the key organisations involved in amphibian and reptile conservation and build partnerships with other organisations that can contribute to recording and monitoring schemes.

Discussion: There was consensus that the partnership landscape should extend to include other wildlife and conservation organisations. There is a need to map out this landscape to identify who these stakeholders could be. This should include community partners, e.g. churches, schools. 
2) Maintain and, where possible, expand the existing surveillance and monitoring schemes and improve coverage.

3) Review the distribution of active volunteers involved in amphibian and reptile recording with a view to targeting areas with poor such representation for future training in identification and monitoring.

Discussion: With reference to priorities 2 and 3, participants discussed shared experiences around the difficulties in recruiting and maintaining a large enough cohort of volunteers across the required geographical range. This can lead to "volunteer fatigue", where a minority of volunteers may be undertaking significant amounts of monitoring, often across a range of projects and priorities. Potential new sources of volunteers were explored, including targeting clubs and groups of people who regularly access the underserved geographical locations, e.g. hill walking clubs, rambling groups. This would require training and/or tools to make monitoring and recording easy and accessible, e.g. Froglife's Dragons on the Hills app. This raised the question as to who would be responsible for the delivery of the monitoring schemes and a reflection on whether schemes could be expanded to include monitoring for disease and genetic bottlenecks.

4) Ensure that all data collected through monitoring schemes are accessible via Scotland's Environment Web (SEWeb).

Discussion: It was noted that there are ongoing discussions around the creation of a central biological recording system which will be widely accessible. Concern was voiced around information on sensitive species being available publicly.

5) In light of concerns about adder populations in Scotland, clarify the status of the species and assess any changes since 1994 .

6) Clarify the status of grass snakes (Natrix natrix) in Scotland and their likely provenance.

7) Expand the use of eDNA sampling in great crested newt (Triturus cristatus) surveillance to provide reliable trend information for Scotland, and trial the use of meta-barcoding eDNA analysis to enable the inclusion of additional species in the surveillance.

Discussion: The method is expensive (£100-£200 per sample) and requires a standardisation of techniques. To support this priority, funding may be available from ScotRail for biodiversity schemes near railway lines. Reference sequences are available on public databases, e.g. Genbank.

Additional comments: There is an untapped resource from surveys carried out by industry and private companies, which is currently not fed into the biological records centres. These, however, are too often regarded as commercially sensitive and not made available.

\section{ACKNOWLEDGEMENTS}

The following are thanked for acting as facilitators/scribes: Tie Caribe, Iain Hill, Amber Mathie, Erik Paterson and Chloe Rossi. The conference organisers are grateful to Rob Raynor for agreeing to present the draft SNH Strategy.

\section{REFERENCES}

ARG UK. (2017). Amphibian Disease Precautions, a Guide for UK Fieldworkers. Advice note 4 (revised). https://www.arguk.org/infoadvice/advice-notes/324-advice-note-4amphibian-disease-precautions-a-guide-for-ukfieldworkers-pdf-2

Nature Back From The Brink. (2018). Roots of Rockingham Project. www.naturebftb.co.uk/theprojects/roots-of-rockingham

Scottish Government. (2012). Non-Native Species: Code of Practice. https://beta.gov.scot/publications/non-nativespecies-code-practice/

Scottish Natural Heritage (SNH). (2017). Pollinator Strategy 2017-2027. https://www.nature.scot/pollinator-strategy2017-2027 